\title{
PRECISE ORTHO IMAGERY AS THE SOURCE FOR AUTHORITATIVE AIRPORT MAPPING
}

\author{
H. Howard ${ }^{\text {a }}$, P. Hummel ${ }^{\text {b }}$
}

\author{
${ }^{\text {a } C o m p a s s D a t a, ~ I n c, ~ C e n t e n n i a l, ~ C o l o r a d o, ~ U n i t e d ~ S t a t e s ~ o f ~ A m e r i c a ~-~ h a y d e n h @ ~ c o m p a s s d a t a i n c . c o m ~}$ \\ ${ }^{\mathrm{b}}$ CompassData, Inc, Centennial, Colorado, United States of America - phummel@ compassdatainc.com
}

KEY WORDS: Aviation, Mapping, Aerodrome Mapping Database, AMDB, Authoritative Map Data, Satellite Imagery, Ground Truth, Ground Control Point, GCP, Feature Extraction, DO-200A, Certified Dataset, Electronic Flight Bag

\begin{abstract}
:
As the aviation industry moves from paper maps and charts to the digital cockpit and electronic flight bag, producers of these products need current and accurate data to ensure flight safety. FAA (Federal Aviation Administration) and ICAO (International Civil Aviation Organization) require certified suppliers to follow a defined protocol to produce authoritative map data for the aerodrome. Typical airport maps have been produced to meet $5 \mathrm{~m}$ accuracy requirements. The new digital aviation world is moving to $1 \mathrm{~m}$ accuracy maps to provide better situational awareness on the aerodrome. The commercial availability of $0.5 \mathrm{~m}$ satellite imagery combined with accurate ground control is enabling the production of avionics certified $.85 \mathrm{~m}$ orthophotos of airports around the globe. CompassData maintains an archive of over 400+ airports as source data to support producers of $1 \mathrm{~m}$ certified Aerodrome Mapping Database (AMDB) critical to flight safety and automated situational awareness. CompassData is a DO200A certified supplier of authoritative orthoimagery and attendees will learn how to utilize current airport imagery to build digital aviation mapping products.
\end{abstract}

\section{INTRODUCTION}

Maps, charts, elevation models are all obvious requirements for a safe and efficient air transportation. Regulated airspace requires routes to be plotted and aircrafts to be tracked in the air between airports on ascent, decent, and on the ground of airports. Airport operations manages the interaction of passenger airplanes, freight planes, fuelling trucks, baggage carts, mechanics and emergency vehicles on a busy airport like Frankfurt am Main, Germany with about 160,000 passengers per day.

In this article the reader is introduced to the standardized mapping effort of airports around the world. The mapping product is referred to as Airport Mapping Database (AMDB).

The purpose of the AMDB is to show an airport in digital form to pilots, operations on the airport site, and the traffic controllers in the tower. The evolution from a paper map to a $1 \mathrm{~m}$ accurate electronic map enables sharing of an interactive common operational picture to support situational awareness for airport operations.

The production of high accurate digital AMDBs requires orthoimages, digital terrain models (DTMs), and ground control points (GCPs). With the support of GIS software, these datasets are combined to build airport mapping products. The product is built in accordance with FAA and ICOA (jointly referred to as Agency) requirements by suppliers that have undergone a rigorous certification process to insure accuracy, consistency and completeness.

\section{AIRPORT MAPPING}

The global airport network is comprised of small areas of land separated by vast geography crossing oceans and international borders. Authoritative mapping data is required for avionics grade mapping data to ensure global flight safety. Agencies require developers of avionics grade map data to be certified to standards such as D0-200A and D0-272.

Ideally each airport map provides the user with a standard set of symbols, termini, units and coordinate systems. Map projections, coordinate systems, units, geodetic datum systems are a complicated challenge to standardize since countries around the world can define these differently. Often the term WGS84 is used without the consideration of national refinements like NAD83, ETRF89, and GDA. An international ITRF2008 datum is refined by a particular epoch or point in time. If not treated correctly these variations cause significant geometric differences from airport to airport.

The choice of geodetic datum, projections and units must be considered the first step when generating authoritative avionic grade mapping data to meet Agency requirements. The same high standards apply when selecting satellite imagery, imagery processing algorithms and mapping extraction methods to build the airport maps. Certified suppliers must qualify their processes and tools used for fixed workflows designed to meet Agency specifications.

FAA and ICAO set mapping data standards for production and documentation that many avionics manufactures and airport operators endorse. Organizations such as the International Air Transport Association (ITATA) work with these Agencies to help formulate industry policy on critical aviation issues. Evolution of these policies and specifications take years and have difficulty keeping pace with rapidly developing technology. Advances in satellite imagery, imagery processing systems and GNSS processing tools enable certified suppliers of airport mapping data to build more accurate and current data to meet Agency requirements.

Key components of airport mapping are imagery and surveyed ground control. These avionics grade data require standardized processes to meet defined data quality requirements that are monitored to ensure compliance and air safety. 


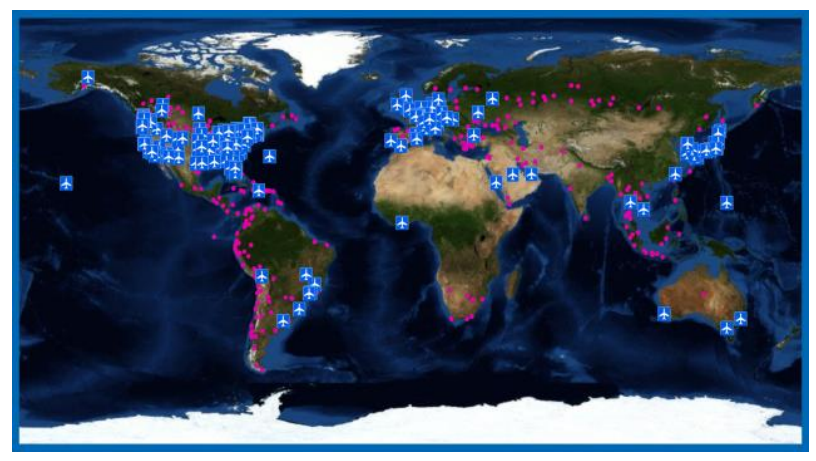

Figure 1: CompassData certified airport images and certified GCPs

\subsection{GROUND TRUTH}

Ground truth of geospatial products is established by survey on the ground with GNSS systems. Photogrammetry and remote sensing rely on photo-identifiable points with exact coordinates on the ground that also are depicted in the imagery acquired either by aircrafts or satellites.

The geodetic processing determines the datum of the coordinate system used for the coordinates for the GCPs. The GCPs should be three time more accurate than the final imagery product being produced $(30 \mathrm{~cm}$ GCP for $1 \mathrm{~m}$ image) to geo-reference the imagery. The GNSS processing has to uniformly provide a geodetic datum and projected coordinate system to match the georeference for the imagery.

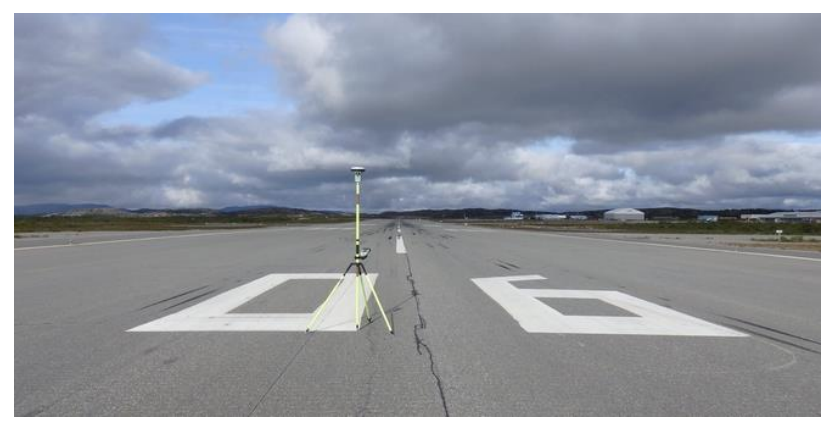

Figure 2: Ground Control Point (GCP) collection on the Airport

\subsection{IMAGERY AND IMAGE-PROCESSING}

High resolution satellite imagery from Digital Globe's satellites or Airbus' satellites are currently meeting the requirements for accurate airport mapping. Ground sample distance, radiometric resolution, and image geometry are the key elements for usable input data for later feature extractions. Alternatively, aerial imagery has the high resolution, clarity and geometric integrity to meet aviation requirements. However, it lacks in standardized global availability compared to the global consistency of satellite imagery.

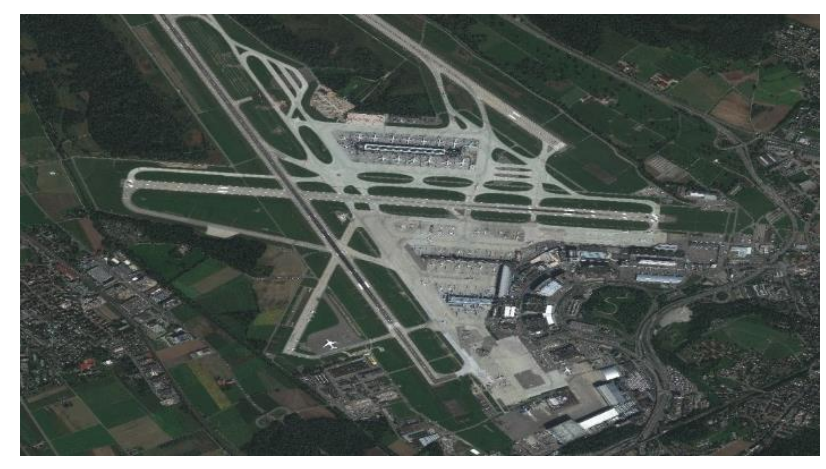

Figure 3: Ortho-Image of an Airport based on Satellite Imagery

Typically, stereoscopic imagery is obtained from a satellite provider. This imagery is accompanied by a sensor model, which is read by photogrammetric software for rough geolocation of the imagery. Relative triangulation is first used to refine the photogrammetric solution and reduce errors between the two images. This corrects errors related to image space but not ground space. Absolute triangulation is then used to adjust the images to ground control, which projects them to their correct real world locations.

A Digital Elevation Model (DEM) is created using the refined stereoscopic imagery using image correlation. By default, this creates a Digital Surface Model (DSM). However, algorithms within the software allow for the creation of a bare earth Digital Terrain Model (DTM) by automatically identifying and removing above ground features. Additional editing is usually required to remove aberrations in the data and structures that were not automatically removed.

An orthorectified image is then created using the edited DTM and one of the refined images. Alternatively, a monoscopic image can be used after processing absolute triangulation. The orthorectified image is thoroughly checked to ensure there are no areas that are warped, due to irregularities in the DTM.

The final orthoimage is then verified through DO-200A accuracy testing. CompassAA, a DO-200A qualified accuracy verification tool, is used to compare the orthoimage to ground control points, which are independent of the image processing. This generates a statistical report to determine pass or fail of the imagery to meet DO-200 requirements. The certified image is then qualified to be used as source data to extract planimetric features for AMDB or other authoritative airport mapping products.

\subsection{AMBD EXTRACTED DATA ELEMENTS}

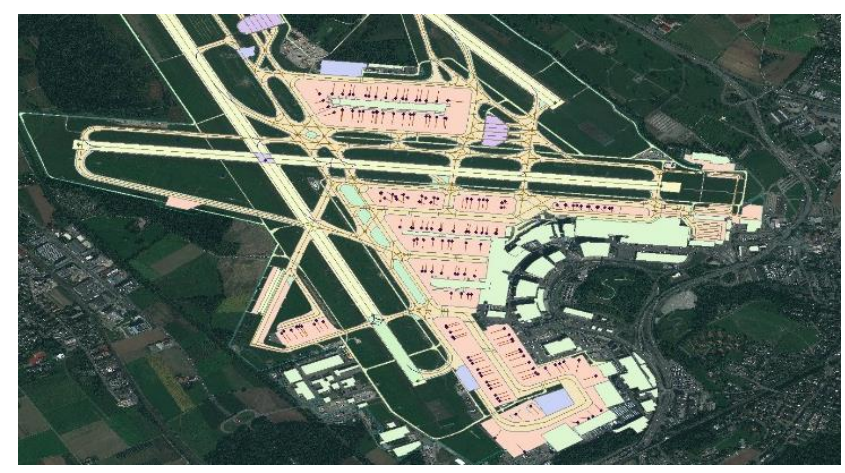

Figure 4: Extracted Features superimposed on Ortho-Image

A DO-200A certified image of the airport, delivered per the developer's data quality requirement, is used as source data for 
feature extraction. The standard defined by DO-272 for AMDB requires the following features to be graphically displayed:

Apron Element, Arresting Gear Location, Arresting System Location, AsrnEdge, AsrnNode, Blast Pad, Construction Area, Deicing Area, Final Approach and Take Off Area, Frequency Area, Helipad Threshold, Hotspot, Land and Hold Short Operation Location, Painted Centerline, Parking Stand Area, Parking Stand Location, Runway Displaced Area, Runway Element, Runway Exit Line, Runway Intersection, Runway Marking, Runway Shoulder, Runway Threshold, Service Road, Stand Guidance Line, Stopway, etc.

The AMDB or other aviation mapping product is verified per the DO-272 requirements for accuracy and currency before being released for use in avionics applications.

\subsection{AVAILABILTY AND CURRENCY}

For every airport map a complete and updated reflection of the real world is required to meet Agency certification requirements. As airports are constantly under construction for extensions and improvements, maintenance of the source data archive of airports is critical to ensure safety. Using current imagery derived from satellite sensors and previously surveyed ground control points, a schedule of maintenance is designed. The use of commercially available imagery sources and certified ground control enable quick delivery of the certified source imagery needed to produce and deploy aviation grade mapping products to pilots and operations.

\section{ICAO AND FAA}

The United States is a member state of the International Civil Aviation Organization (ICAO). The International Civil Aviation Organization (ICAO) is a UN specialized agency, established by Countries in 1944 to manage the administration and governance of the Convention on International Civil Aviation (Chicago Convention). CompassData, Inc. received a Letter of Acceptance (LOA) from the US Federal Aviation Administration (FAA) that acknowledges the compliance of our ground control and imagery as source data for Airport Map Databases (AMDB). CompassData products and processes meet the rigorous requirements of FAA Advisory Circular 20-153A "Acceptance of Aeronautical Data Processes and Associated Databases" and RTCA Inc. (Radio Technical Commission for Aeronautics) document DO-200A, "Standards for Processing Aeronautical Data". RTCA/DO-200A is the standard the Agencies recognizes for the processing, quality assurance, and data quality management of aeronautical data.

The DO-200A certification is recognized globally by suppliers of avionics products to meet ICAO and FAA requirements. CompassData's Certification for key components of the airport mapping process is unique in the profession. Off the shelf Certified GCPs and imagery enables airport map providers a path to produce certified aviation data without the costly and time consuming efforts required to develop and certify process that are not part of the organizations core competency. To supply data to support global avionics standards mapping organizations must meet these standards. The data quality is defined between users and providers by several characteristics including accuracy, resolution, integrity (referred to as assurance level), timeliness, completeness, traceability and format. Adopters of Certified GCP and imagery data are also guaranteed a source of current data on a maintenance plan to meet Agency requirements.
CompassData was awarded an FAA Type 1 Letter of Acceptance (LOA006DE) in 2014. CompassData has been a data provider of Ground Control Points to the geospatial and aviation market since 1996. As the aviation market moved to a digital format for AMDB and Airport Moving Map, CompassData identified the need for certified GCP and imagery data to support suppliers of aviation grade systems to meet growing demand. CompassData's LOA with the FAA is based on the processes to supply and maintain highly accurate and consistent GCPs and imagery as a key component to support the production of authoritative airport mapping.

\subsection{CERTIFICATION OF AN ORTHO-IMAGE}

The created ortho-imagery that is the base for feature extraction is certified through image accuracy verification. For the process, independent GCPs are superimposed onto the ortho images and residuals captured by the CompassAA ${ }^{\mathrm{TM}}$ software. The certified verification process is conducted to the specification defined by the data quality requirement for the individual product. Compass $\mathrm{AA}^{\mathrm{TM}}$ is certified as a software tool to determine pass or fail for the imagery to support final delivery.

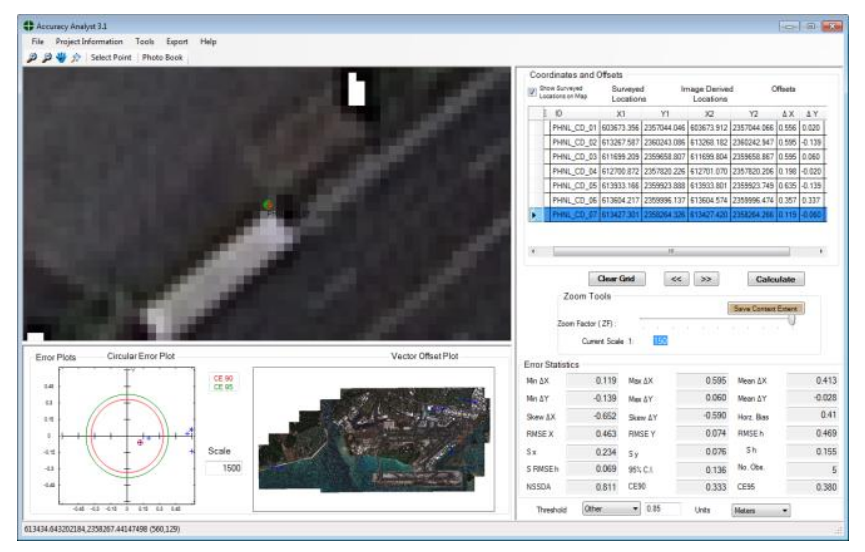

Figure 5: Verification with CompassAA Software

\section{DIGITAL MAP FOR SITUATONAL AWARENESS}

Digital mapping of airports is leveraging GIS technology to support the extraction of different feature layers from remotely sensed imagery. Development of a common operational picture for the airport can be used to support moving map displays for operational activities. Live events like weather, service vehicles and snowplow telematics can be overlaid on the display to support real time decisions. For pilots and equipment operators these maps can be reduced to base maps to display other real time moving participants as a dynamic overlay raising the situational awareness.

\subsection{CERTIFICATION DO-200A BY FAA}




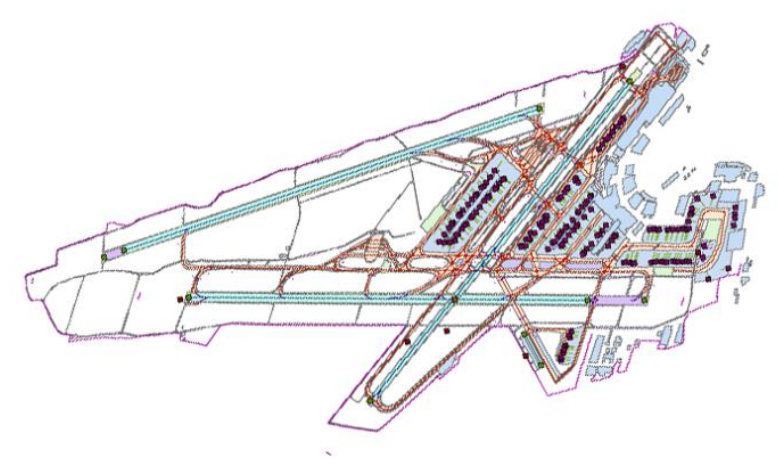

Figure 6: GIS layers of an airport

Three-dimensional maps and photorealistic maps of airports supports decisions for security, operations and traffic controllers. The availability of authoritative airport mapping data is empowering the development of application that automate operations of the airports improving safety and efficiency to support the rapid expansion of commercial, business and freight aviation.

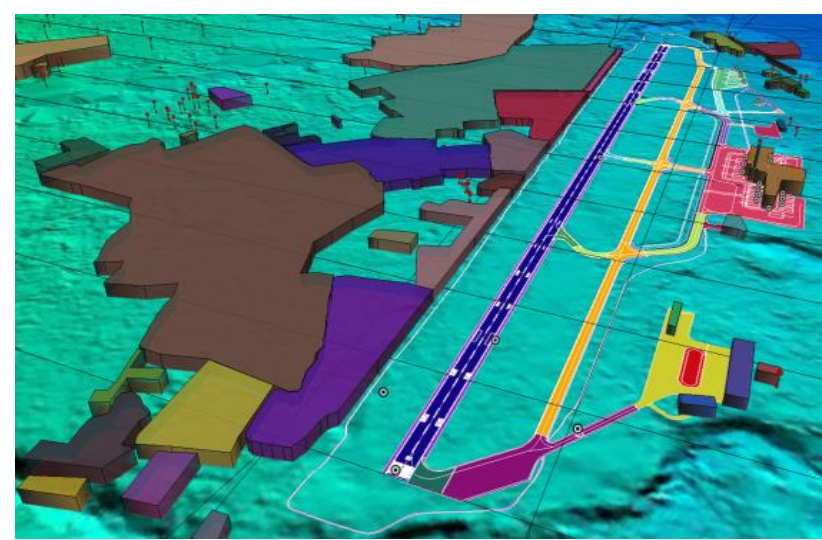

Figure 7: Three-dimensional airport map

\section{CONCLUSIONS}

Electronic airport maps deployed in today's avionics platforms are driving the requirements for $1 \mathrm{~m}$ accurate and current remotely sensed airport imagery. Commercial carriers and private operators still rely on the hardcopy prints of airport and aeronautical charts to be compliant with current regulations. Updates and maintenance to paper maps is cumbersome and real time situational awareness is not available. Many pilot carry digital data on a mobile device with paper maps as required. The mobile device cannot be connected to the airframe today unless the hardware and digital data is certified to ICOA or FAA standards. Certified accurate GCP, imagery and airports maps are key components as the digital cockpit and digital flight bag become standard operating procedure. Authoritative $1 \mathrm{~m}$ mapping data integrated with navigation systems that use GNSS technology, signal beacons, VOR stations and other telematics will create a common operational picture to support airport operations and safety.

Aeronautical chart and AMDB data has to meet Agency standards and also be certified for completeness and accuracy. CompassData provides certified sources of current and accurate remotely sensed imagery to empower avionics suppliers to deploy digital systems to meet the demand for next generation air transportation.

\section{ACKNOLEDGEMENTS}

Figure 4 and 6 are a courtesy of Performance Software.

\section{REFERENCES}

Press Release: CompassData, Inc., Certified by FAA to Collect and Verify Data for Aviation Mapping

Centennial, Colorado, 14 April 2014

Press Release: CompassData, Inc., Granted ISO 9001:2008 Certification for Quality Management Systems

Centennial, Colorado, 5 February 2015

Website of International Civil Aviation Organization (ICAO), titled About ICAO, http://www.icao.int/abouticao/Pages/default.aspx

Website of Federal Aviation Administration (FAA) titled Mission Statement, 09/20/2010, http://www.faa.gov/about/mission/

FAA Advisory Circular AC 20-153A, http://www.faa.gov/documentLibrary/media/Advisory_Circular/ AC_20-153A.pdf 
The International Archives of the Photogrammetry, Remote Sensing and Spatial Information Sciences, Volume XLI-B8, 2016 XXIII ISPRS Congress, 12-19 July 2016, Prague, Czech Republic 